\title{
An Introduction to the Multidimensional Real-Time Economic Modeling
}

\author{
Mario Arturo Ruiz Estrada', Vgr Chandran'1, Muhammad Tahir ${ }^{2}$
}

ABSTRACT This article proposes a new economic modeling theoretical framework known as multidimensional
real-time economic modeling (MRTE-Modeling). The model is an important tool that economists and
educators can use to demonstrate the multidimensional aspects of economic behavior. MRTE-Mod-
eling facilitates the analysis of a series of complex and dynamic economic problems that can affect
market behavior from a multidimensional perspective. The proposed MRTE-Modeling framework is
based on the uses of an alternative mathematical modeling framework, multidimensional graphi-
cal modeling approach, and computer algorithm and, thus, allows the possibility to transition from
2-dimensional economic dynamic modeling to multidimensional real-time economic modeling.
Therefore, the main objective of using the proposed experimental model in the field of economics
is to analyze different macroeconomic scenarios to monitor and provide a warning of possible unex-
pected economic failure(s). The proposed alternative experimental model is based on the application
of Econographicology. Hence, our model is expected to offer policy makers and researchers new
analytical tools to study the impact and trend of economic failures in the economy of any country
from a new perspective.

KEY WORDS: economic modeling, real-time analysis, multidimensional coordinate spaces, econographicology

JEL Classification: Y20

'Department of Economics, Faculty of Economics and Administration, University of Malaya, Malaysia 2Department of Management Sciences, COMSATS Institute of Information Technology, Pakistan

\section{Introduction}

This paper aims to illustrate an alternative economic modeling framework to study market behavior as a whole. It is based on the use of a new multidimensional graphical modeling technique coupled with the application of a real-time effect. The proposed modeling framework builds a computer algorithm that can facilitate the input, storage, classification, analysis and visualization of a large number of endogenous vari-

\section{-}

Correspondence concerning this article should be addressed to: Mario Arturo Ruiz Estrada, Department of Economics, Faculty of Economics and Administration, University of Malaya, Kuala Lumpur 50603, Tel (+60) 37967-3728, E-mail: marioruiz@um.edu.my ables that can simultaneously affect market behavior. For a long time, academics and scholars depended on the application of 2-dimensional graphical modeling, and now it is time to transition to multidimensional graphical methods given the advancement in computer technology. The simple graphical format of 2-dimensional modeling can show only the visualization of historical and forecasting data in the same graphical space within a limited space of time. However, multidimensional real-time graphical modeling can help researchers observe all possible changes in a large number of variables. The changes in all these variables allow learners to clearly observe the cause and effect of different economic phenomena through the visu- 
alization of multidimensional graphs that permit the observation of a large number of variables in real time. Basically, the transition from 2-dimensional graphical modeling to multidimensional real-time graphical modeling requires researchers to learn how to plot and draw multidimensional graphs using different multidimensional coordinate spaces. Nevertheless, the adaptability to transition from static 2-dimensional graphical modeling to multidimensional real-time graphical modeling takes time. In economic analysis, real economic problems can be identified and incorporated into a multidimensional graph to visualize the movements of variables as simulations of economic variables. Multidimensional graphs can also be used to describe dynamic and multi-functional analyses that represent changes within the total function. Furthermore, multidimensional graphs can also help researchers visualize the changes in all exogenous variables simultaneously.

We suggest the application of Econographication, pioneered by Ruiz Estrada (2007), to learn and understand multidimensional graphs under the application of multidimensional coordinate spaces. Ruiz Estrada contended that the teaching and learning of multidimensional graphs require basic knowledge about the 2-dimensional Cartesian coordinate system, which serves as the foundation in plotting multidimensional graphs in economics. Multidimensional graphs can be constructed in five basic steps: first, the acquisition of basic knowledge of plotting and drawing 2-dimensional graphs in the Cartesian coordinate system; second, the acquisition of basic knowledge of Euler's geometry; third, the attainment of a basic idea of time and space in the graphical space; fourth, the construction of solid prototype(s) using basic materials such as wood, papers, plastics and others; and fifth, the adaptation to learn and understand different dimensions that exist in multidimensional graphs. Before proceeding further, it should be noted that the Omnia Mobilis assumption of Ruiz Estrada (2011a) must be applied to multidimensional graphs to generate the environment of freedom in different dimensions needed for the graphs to work without any restriction or condition. The Omnia Mobilis assumption suggests applying economic modeling in real time to observe the changes in all variables simultaneously. The Omnia Mobilis assumption opens a new era in economic theory by applying multidimensional graphs and real-time multidimensional graphical modeling. We can say that the contribution of the Ceteris Paribus assumption was great in its time, but in the present time, it is not sufficient to simply explain complex and dynamic economic phenomena. The difference between the Ceteris paribus and $\mathbf{O m}$ nia Mobilis assumptions is that the Ceteris Paribus assumption takes only a snapshot of the historical moments of some economic phenomena at one time, whereas the Omnia Mobilis assumption shows video running in real time. The Omnia Mobilis assumption (everything is moving) was introduced to reduce dependency in the use of the ceteris paribus assumption to explain an economic phenomenon. We propose the openness of substantive isolation through the application of the Omnia Mobilis assumption. Hence, the use of the ceteris paribus assumption is minimal and even relaxed in the application of multidimensional graphical modeling. The Omnia Mobilis is in contrast to the process of building economic models to explain a complex economic phenomenon in $2 \mathrm{di}$ mensions that begins with the ceteris paribus assumption. Marshal (1890) clearly articulated the use of the ceteris paribus assumption in economic models. According to Marshall (1890, p. 304),

"The element of time is a chief cause of those difficulties in economic investigations which make it necessary for man with his limited powers to go step by step; breaking up a complex question, studying one bit at a time, and at last combining his partial solutions into a more or less complete solution of the whole riddle. In breaking it up, he segregates those disturbing causes, whose wanderings happen to be inconvenient, for the time in a pound called Ceteris Paribus. The study of some group of tendencies is isolated by the assumption other things being equal: the existence of other tendencies is not denied, but their disturbing effect is neglected for a time. The more the issue is thus narrowed, the more exactly can it be handled: but also the less closely does it correspond to real life. Each exact and firm handling of a narrow issue, however, helps towards treating broader issues, in which that narrow issue is contained, more exactly than would otherwise have been possible." 
Marshall's (1890) approach allows the analyses of complex economic phenomena in parts such that each part of the economic model can be joined to generate an approximation of the real world. This approach can be termed as the Isolation Approach, and according to Marshall (1890) and Schlicht (1985), it originates from two possible isolation clauses. First, the ceteris paribus assumption allows some variables to be considered unimportant. This clause is called substantive isolation. Substantive isolation considers that some unimportant variables cannot significantly influence the final result of the economic model. Second, the ceteris paribus assumption allows the impact of some important factors to be disregarded. In this case, the application of the ceteris paribus assumption is purely hypothetical; therefore, the second clause is called hypothetical isolation. It allows parts of the model to be managed more easily and efficiently. The goal is, therefore, to achieve a parsimonious model that is able to explain the complex real world. The idea is to draw multidimensional graphs that can aid in the visualization of a large number of variables in different spaces on the same graphical space. However, beyond that, multidimensional graphs provide an alternative to Marshall's view of a step-by-step cumulative partial approach to modeling a complex economic phenomenon. To apply the multidimensional real-time economic modeling framework, we generate an effect of movement live or in real time. Hence, the basic premise of the multidimensional real-time graphical modeling framework assumes that markets are in a constant dynamic imbalanced state that experiences constant chaos and high vulnerability. Furthermore, the concept of equilibrium in the multidimensional real-time economic modeling framework is applied to the analysis of market failures. Equilibrium is considered as a leak in the momentum of balance among all variables that can appear at any time. However, we cannot predict exactly when this synchronized balance is going to appear. In the multidimensional real-time economic modeling framework, there is not even a single endogenous variable or single exogenous variable available. Multidimensional real-time economic modeling allows for the observation of a multidimensional graph that is moving different variables in real time in the same graphical space. This multidimensional graph is also capable of showing the regular and irregular behaviors of market failures in the short run. Lastly, multidimensional realtime economic modeling is based on the use of multidimensional partial differentiation.

\section{Law of demand and supply from a multidimensional perspective}

Typically, the study of market behavior under the demand and supply law is graphically represented by the traditional demand and supply curves embodied in two-dimensional graphs. In the case of the demand and supply curves in multidimensional form, the Dynamic Imbalanced State (DIS) (Ruiz \& Yap, 2013) must be used under the Omnia Mobilis assumption. The DIS permits the demand and supply analysis, which includes internalities and externalities such as economic factors, social factors, political factors, technological factors, environmental factors, institutional factors and other different economic agents and institutional behaviors (e.g., public sectorgovernment, private sector-firms, consumers-households) to explain the real behavior of the market in its whole context. Regarding the application of the analytical graphical method in economics, it is necessary to mention the major contribution of Merritt (1838). Cournot derived the first formula for the rule of supply and demand as a function of price. He was also the first economist to draw supply and demand curves on a graph. This author believed that economists should utilize graphs only to establish probable limits and express less stable facts in more absolute terms. He further held the view that the practical use of mathematics in economics involves not only strict numerical precision but also graphical visualization. In fact, Cournot suggested the application of the first quadrant of the 2-dimensional Cartesian co-ordinate system by drawing two linear functions to represent demand and supply curves in the same graphical space. In addition to Cournot, other innovative economists who contributed to the analytical graph systems in economics over time include Leon Walras, Vilfredo Pareto, Alfred Marshall and Francis Ysidro Edgeworth (McClelland, 1975).

The reason behind applying the demand and supply law to the study of market behavior is to find market equilibrium among a large number of possible combinations between two specific variables followed by the price of the commodity (exogenous variable) and 
quantity (endogenous variable) in a fixed period of time and space. The mythical part here is how different economic agents (buyers and sellers) react or overreact unconditionally according to the effect of the unexpected behavior of price commodity on the quantity demanded and supplied.

The main reason to visualize demand and supply through the implementation of multidimensional real-time graphical modeling is to explore unknown dimensions of market behavior from a multidimensional perspective. The basic premise is that the market undergoes constant evolution across time and space. This means that markets in each period of history can show different structures, internalities, externalities, institutions, and economic agents can show different behaviors. Hence, the study of market behavior requires alternative economic assumptions and economic models based on each specific period of history. Additionally, we shed some light on the unexpected, irrational and trends that market behavior can experience at any time from a multidimensional perspective. Therefore, the study of market behavior becomes more complicated, demonstrating with low levels of accuracy across the time dimension. In terms of classification, market equilibrium can be either partial or general. Furthermore, partial market equilibrium can be divided into linear and non-linear modeling from the mathematical and graphical perspectives. Basically, partial market equilibrium using the linear modeling approach is based on the analysis of a single commodity and three variables such as the quantity demanded $\left(\mathrm{Q}_{\mathrm{d}}\right)$, quantity supplied $\left(\mathrm{Q}_{\mathrm{s}}\right)$ and the price of the commodity (P). This specific model is represented by two linear equations. The main characteristic of the demand linear equation (see expression 1 ) is that its slope is always negative (downward) because price and quantity demanded move in opposite directions. The slope of the supply linear equation (see expression 2) is positive (upward) because the price and quantity move in the same direction. Furthermore, the price commodity equilibrium $\left(\mathrm{P}^{*}\right)$ is a result of the elimination of variables from the identity $\mathrm{Q}_{\mathrm{d}}=\mathrm{Q}_{\mathrm{s}}$ (see expression 3). Additionally, the quantity equilibrium $\left(Q^{*}\right)$ is a result of replacing the price equilibrium $\left(\mathrm{P}^{*}\right)$ with the quantity demanded equation $\left(Q_{d}\right)$ (see expression 4). Finally, market equilibrium $(\varepsilon)$ is represented by expression 5 .

$$
\begin{aligned}
& Q_{d}=\alpha-\beta P \\
& Q_{s}=-\pi+\lambda P \\
& Q_{d}=Q_{s}=>P^{*} \\
& Q^{*}=Q_{d}=\alpha-\beta P^{*} \\
& \varepsilon=\left(P^{*}, Q^{*}\right)
\end{aligned}
$$

Partial market equilibrium by non-linear modeling is represented by the application of a linear equation (see expression 7) and a non-linear equation (see expression 6). In the first example, we need to identify the price equation by constructing a single equation (see expression 8). Subsequently, we proceed to find the price commodity equilibrium $\left(\mathrm{P}^{*}\right)$. It is based on the application of the quadratic formula (see expression 9) to the single equation or price equation. Hence, we consider only the positive final value from the quadratic function because only positive values are economically admissible. The last step is to incorporate the price commodity equilibrium $\left(\mathrm{P}^{*}\right)$ into the quantity demanded function $\left(Q_{d}\right)$ to find the obtained equilibrium quantity $\left(\mathrm{Q}^{*}\right)$, as shown in expression 10 . The final equilibrium is presented in equation 11 .

$\mathrm{Q}_{\mathrm{d}}=\alpha-\mathrm{P}^{2}$

$\mathrm{Q}_{\mathrm{s}}=\lambda \mathrm{P}-\pi$

$f(\mathrm{P})=\mathrm{Q}_{\mathrm{d}}+\mathrm{Q}_{\mathrm{s}}$

$\mathrm{P}_{1}{ }^{*}, \mathrm{P}_{2}{ }^{*}=\frac{-\mathrm{b} \pm\left(\mathrm{b}^{2}-4 \mathrm{ac}\right)^{1 / 2}}{2 \mathrm{a}}=>\mathrm{P}_{1}^{*}, \mathrm{P}_{2}^{*} \cap \mathrm{R}_{+}$

$\mathrm{Q}^{*}=\mathrm{Qd}=\mathrm{Q}_{\mathrm{d}}=\alpha-\left(\mathrm{P}^{*}\right)^{2}$

$\varepsilon=\left(\mathrm{P}^{*}, \mathrm{Q}^{*}\right)$

The analysis between static, dynamic and real time plays an important role in the current era of economic modeling. Initially, we can say that economic modeling in real time does not represent a simple relationship between two variables such as a dependent variable and independent variable(s) that are fixed in a specific period of time. In our case, economic model- 
ing in real time suggests that it is not sufficient to use only static and dynamic economic modeling.

\section{Traditional economic modeling vs. economic modeling in real time}

We find that economic modeling in real time can offer an alternative visual approach to observing the behavior of macroeconomic and microeconomic variables that move through a single screen live. To generate this type of modeling, we need to use advanced information technology and multidimensional graphical modeling. Economic modeling in real time allows us to observe different changes in different variables in the same graphical space. All the variables defined in the abovementioned expressions constantly move through different periods of time (years, months, weeks, days, hours, minutes and seconds) and space (different geographical spaces). It is important to mention that economic modeling in real time is an alternative approach to studying economics from a different perspective. We suggest a major transformation in the economic modeling-oriented framework to detect economic failures in a timely manner. We think that it is very difficult to quantify or predict the behavior of any complex economic phenomenon in the long run because domestic factors and externalities in different economies change at different speeds. It is understood that changes in different factors such as factors in the social, economic, technological and political arenas suffer differently in the different world economies. These fast transformations that economies experience cannot be accurately observed in static and dynamic economic modeling or forecasting. Since Smith's approach in 1776 until today, different schemes of economic modeling such as static and dynamic schemes have been used, as evidenced in a large number of essays, articles and books in libraries, the internet and journals. All these documents have used different theoretical approaches such as descriptive, theoretical, experimental and mathematical frameworks including econometrics. Furthermore, they have given us a general idea about some economic phenomenon or behavior in a specific historical moment through a frozen picture in 2 dimensions (X, Y). To analyze an economic phenomenon, we typically focus on selecting a few variables whose impact is expected to be more visible and ignoring the less important variables floating under the application of "the ceteris paribus assumption" to support possible unexpected scenarios that cannot be simultaneously included in our analysis. It also seems important to highlight here that partial market equilibrium assumes the use of the ceteris paribus assumption. The doctrine here is to isolate the less important variables from the study of market behavior and focus our attention on two specific variables such as the price of the commodity (P) and the quantity (Q). In fact, the price of the commodity $(\mathrm{P})$ is the only variable that can affect quantity. Nevertheless, general market equilibrium is based on the use of a multi-commodity market. The approach is to include more commodities in our analysis, represented by n-equations in the quantity demanded function (see expression 12) and the quantity supply function (see expression 13). Market equilibrium is represented by expression 14. Lastly, it is important to state that general market equilibrium also rests on the Ceteris Paribus assumption.

$$
\begin{aligned}
& \mathrm{Qd}_{\mathrm{i}}=\mathrm{Qd}_{\mathrm{i}}\left(\mathrm{P}_{1}, \mathrm{P}_{2}, \ldots, \mathrm{P}_{\infty}\right) \quad \mathrm{i}=1,2, \ldots \infty \\
& \mathrm{Qs}_{\mathrm{i}}=\mathrm{Qs}_{\mathrm{i}}\left(\mathrm{P}_{1}, \mathrm{P}_{2}, \ldots, \mathrm{P}_{\infty}\right) \quad \mathrm{i}=1,2, \ldots \infty \\
& \varepsilon_{\mathrm{i}}=\left(\mathrm{P}_{1}, \mathrm{P}_{2}, \ldots, \mathrm{P}_{\infty}\right)=0
\end{aligned}
$$

After a short review of the demand and supply model, the following question arises: can the classic analysis of demand and supply fully capture the behavior of the market as a whole? However, we suggest that market behavior needs to be studied based on the interaction of a large number of sub-markets. At the same time, all sub-markets should be kept in a permanent dynamic imbalanced state under the application of the Omnia Mobilis assumption. In fact, the market can be considered as a multidimensional system that interacts and works in perfect harmony without any restriction or condition. Our idea of equilibrium is quite different from the traditional classic view because we view equilibrium as an imbalanced synchronization state that can appear in unexpected and unlimited time. The imbalanced synchronization state is considered a fleeting momentum as a result of the relaxation (or lesser instability) of all sub-markets. It depends on the economic, social, political, technological, and environmental factors; market structures; institutions; and economic agents' behavior. In the same context, 
we suggest the use of the dynamic imbalanced state (DIS), which can help explain possible unexpected market behavior. The dynamic imbalanced state is not a chaotic state; rather, it is the unconditional and unexpected complex sensitive reaction of a large number of sub-markets that is generated by different institutional, economic, political, social, technological, and environmental forces simultaneously under the condition of uncertain non-rational behavior. Therefore, all submarkets do not necessarily need to be in equilibrium simultaneously because all sub-markets are kept in a dynamic imbalanced state. Moreover, all sub-markets can experience an imbalanced synchronization state at any time. It is a short, fleeting transitional state and is unpredictable and spontaneous. Market equilibrium is not a static and isolated phenomenon; it is a transitional and evolutionary state that cannot be controlled and determined arbitrarily. When studying market equilibrium, we must remember that it is not a natural phenomenon that can be exactly measured or demonstrated by the sciences. In our case, market equilibrium can be considered a dynamic imbalanced state, whereby humans' interactions with each other to satisfy their needs always change according to the different periods of history. The analysis of market behavior under the application of the dynamic imbalanced state can be shown by constructing the quantity demanded equilibrium $\left(\mathrm{Q}_{\mathrm{d}}{ }^{*}\right)$ (see expression 15) and quantity supplied equilibrium $\left(\mathrm{Q}_{\mathrm{s}}{ }^{*}\right.$, see expression 16$)$. Hence, the interaction between the $\mathrm{Q}_{\mathrm{d}}{ }^{*}$ and $\mathrm{Q}_{\mathrm{s}}{ }^{*}$ identifies the price commodity equilibrium $\left(\mathrm{P}^{*}\right)$ for any commodity. The measurement of the quantity demanded equilibrium $\left(Q_{d}^{*}\right)$ depends on the interaction of a large number of variables without any restriction(s) or classification (see expression 15), while in the case of partial market equilibrium, only the price commodity $(\mathrm{P})$ can affect the quantity (Q). Therefore, we attempt to account for a large number of variables that can influence the quantity demanded equilibrium $\left(Q_{d}^{*}\right)$.

From a graphical perspective, the quantity demanded equilibrium $\left(\mathrm{Q}_{\mathrm{d}}{ }^{*}\right)$ is the vertical axis among all sub-axes located in the bottom part of each sub-level. All these sub-axes at the bottom part of each sub-level are directly connected to the quantity demanded equilibrium $\left(Q_{d}^{*}\right)$ using straight lines until we can build the demand surface by sub-level, whereby each sub-level represents a specific commodity in the market. A large cylinder can be generated by connecting all sub-levels. It is assumed that each sub-axis in its sub-level shows growth rates $(\Delta)$ in real time $\left(^{*}\right)$, as depicted in Figure 1. Additionally, we must apply the Omnia Mobilis assumption to generate the relaxation of all variables affecting the demanded quantity equilibrium $\left(\mathrm{Q}_{\mathrm{d}}\right)$ because we avoid isolating some of the variables that the Ceteris paribus assumption considers less important. However, if all variables suffer because of relaxation, then the umbrella is open and the price imbalance $(\mathrm{P} \approx)$ becomes lower, according to Figure 2. From a graphical perspective, the price imbalance $(\mathrm{P} \approx)$ is a vertical axis located between the demand and supply surfaces. Figure 2 shows that each commodity has its own specific sub-level (or commodity) and quantity demanded imbalance $(\mathrm{Qd} \approx)$ and quantity supplied imbalance (Qs $\approx$ ) simultaneously in its specific price imbalance $(\mathrm{P} \approx)$.

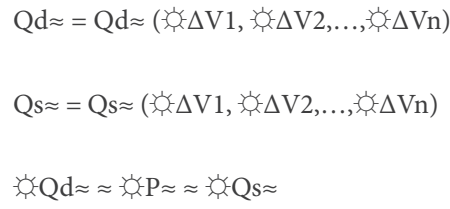

\section{Can we provide an alert or forecast of market failures?}

This section is devoted to the study of market failures from an economic modeling perspective. It seems important to make a clear distinction between economic forecasting modeling and economic alerting modeling. Economic forecasting modeling is based on the classic use of econometrics under the application of different techniques, methods and models using sophisticated software such as SSPS, SAS, STAT, EVIEWS AREMOS, DATADESK and others. The modern econometrics software always adopts a dynamic modeling approach to analyze time-series, cross-sectional and longitudinal data. Forecasting economic modeling attempts to give us some basic and preliminary reasons behind the cause and effect of any economic phenomenon. In addition, we find that econometric models did not precisely predict market failures in the medium and long run with accuracy or a small margin of error until recently.

Furthermore, forecasting economic modeling under the application of econometrics can have low levels of 


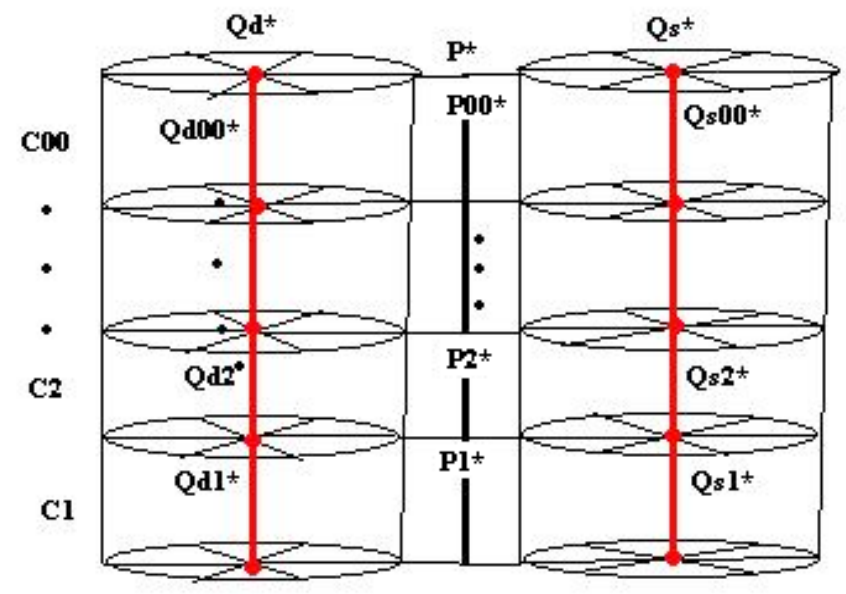

Figure 1. Quantity Demanded Equilibrium (Qd*), Quantity Supplied Equilibrium (Qs*) and Price Commodity Equilibrium (P*) (Ruiz Estrada, Yap, \& Nagaraj, 2008)

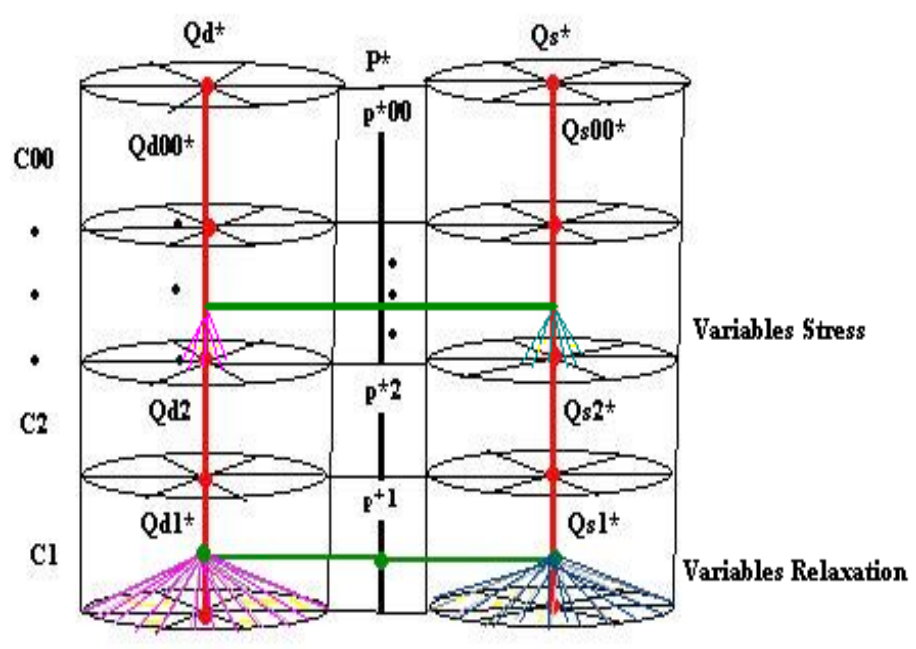

Figure 2. Demand Surface and Supply Surface (Ruiz Estrada et al., 2008)

accuracy and be unclear and unstable scenarios in the long run. Hence, the economic forecasting modeling of market failures in the long run becomes impossible and unrealistic. It can originate from the quickly rising challenges of economic, social, political and technological aspects and institutions in the market. Various factors are responsible for the low level of accuracy in forecasting market failures in the long run. Rapid technological changes, the vulnerability of the world economy due to international trade and investment under the umbrella 
of globalization, and the unpredictability of natural disasters due to a lack of ability can explain why the forecasting economic models are unable to correctly predict economic crisis in the long run.

By contrast, economic alerting modeling is based on mathematical models and computer programming supported by software and dynamic simulators. This type of economic modeling permits us to observe the behavior of the market as a whole in real time without any restrictions or limitations. Hence, the appearance of unexpected market failures because of an oil crisis, a financial crisis, or an international trade crisis become more difficult to predict than in the past, as market failure shows different signs and behaviors in different periods of time in the history of the world economy. Market failures can appear in different periods of time and retain their unique characteristics before the market experiences a substantial full or partial recovery from a deep or superficial crisis. The magnitude of the final impact of market failures can vary across different periods of time and geographical spaces.

More specifically, the negative impact of any market failure can be accounted for by the fast expansion of unemployment, uncontrolled and galloping inflation, constant economic slowdown, rapid and uncontrolled expansion of poverty, low stock market performance, constant currency depreciations, less international trade, high speculation in real estate and consumer uncertainty. Lastly, economic alerting modeling utilizes multidimensional graphical modeling offered by Econographicology and economic modeling in real time.

\section{The idea of time and space in economic analysis}

This section demonstrates that the 2-dimensional space $(\mathrm{X}, \mathrm{Y})$ and 3-dimensional space $(\mathrm{X}, \mathrm{Y}, \mathrm{Z})$ can show only a micro-picture in the visualization of complex and dynamic economic phenomena. The hypothesis of this research paper is that the 2-dimensional and 3-dimensional spaces are micro-slices of the large Mega-space or Universe in a fixed period of time and common space. Therefore, to test the proposed hypothesis, we suggest the application of an alternative multidimensional graphical modeling approach that is called "the Mega-Space Cartesian Plane". For a long time, economists attempted to visualize simple (static) or complex (dynamic) economic phenomena in different periods of time using the same graphical space through the application of 2-dimensional spaces (X, Y) or 3-dimensional spaces (X, Y, Z). The most common dimension applied to economic analysis is the 2-dimensional space $(\mathrm{X}, \mathrm{Y})$ to visualize historical data and explain economic theories and results from economic models under the application of econometrics. For example, the graphical visualization of the behavior of any country in response to any economic phenomenon (G.D.P. rates, inflation rates, exports/imports, etc.) is based on the application of the 2-dimensional Cartesian plane (X, Y). Typically, economists attempt to interpret a group of points located in different places according to time and space. All these points are connected by lines to visualize the historical trends from the past until today or possible results from economic models or forecasting models. In fact, the main idea of applying 2-dimensional spaces (X, Y) to economic models or forecasting models is to visualize how an economic phenomenon behaves under the impact of internal and external factors. Hence, economists can search for suitable policies to prevent possible economic failures or possible results of decisions made in the short or long term.

\section{Why do economists use 2-dimensional and 3-dimensional spaces?}

Economists use 2-dimensional space (X, Y) or sometimes 3-dimensional space (X, Y, Z) in the graphical representation of complex and dynamic economic problems, and it is important to determine the underlying reasons behind this usage. In our opinion, the following reasons can explain the use of these spaces in the study of economics. .

1. These two graphical models were developed and established in Lafleur's (1960) introduction of the 2-dimensional Cartesian plane. Since then, the application of the 2-dimensional space in economic graphical analysis has been a "tradition".

2. The 2-dimensional space is "easy to apply" when visualizing basic trends or values in the same graphical space. We randomly selected approximately 5,000 documents (JSTOR and DIRECT SCIENCES -ELSEVIER-, 2013) from different reputable journals and text books in economics, econometrics, business, statistic, mathematic and 
sciences. Of all these documents $99.50 \%$ used 2-dimensional spaces (X, Y) and only $0.05 \%$ used 3-dimensional spaces (X, Y, Z).

3. The logical explanation for the frequent use of 2-dimensional spaces is the ease of plotting, drawing and visualizing any economic phenomenon. Therefore, 2-dimensional spaces can generate a clear visual and mental refraction to graphically present complex and dynamic economic phenomena in the same space and time.

4. It is difficult to find "alternative and suitable multidimensional graphical models" to move from 2-dimensional space graphical modeling to graphical modeling of multidimensional spaces. This research paper encountered some difficulties in generating this crucial visual and mental transition from 2-dimensional spaces to multidimensional spaces.

The main consideration of this research paper is that 2-dimensional and 3-dimensional spaces are not able to capture the behavior of the real economic world as a whole. It is understood that the real world is changing constantly and in different spaces and across time. To test this proposition, we assume that inside the MegaSpace or Universe, the number of General-Spaces, Sub-Spaces, Micro-Spaces, Nano-Spaces and JI-Spaces is infinite. All these spaces move differently. The logic behind the plotted points on the 2-dimensional or 3 -dimensional Cartesian plane in this research is quite different from the traditional view because a single point plotted on the Cartesian plane is considered a rigid body. The first assumption applied in this research paper is that two rigid bodies cannot occupy the same space simultaneously. Different rigid bodies unfold and contend for different positions in the Mega-Space. The rigid bodies constantly move and generate different graphical reflections. The second assumption is that different rigid bodies in the MegaSpace, General-Spaces, Sub-Spaces, Micro-Spaces, Nano-Spaces and JI-Spaces run at different speeds of time. We assume that there are different types of time and that these times are followed by general times, partial times and constant times. The general time runs in the Mega-Space, but the General-Spaces, Sub-Spaces, Micro-Spaces, Nano-Spaces run under different partial times (see Figure 3). The JI-Spaces runs in constant time. The rigid body hangs on a specific point of the
Mega-Space. The rigid body in the economic analysis can be represented by the interaction among fixed values in some specific space through the Cartesian plane. These values can be classified by absolute values (e.g., amounts of money or units of goods) and relative values (e.g., growth rates or percentages). In addition, it is also important to mention the roles of Euclidian geometry and Minkowski's 4-dimensional spaces (Einstein, 1961) in supporting our proposition. Einstein used both geometrical models to explain his theory of relativity. These two geometrical modeling frameworks are strongly supported by his formal mathematical and theoretical frameworks. However, these two geometrical modeling frameworks are not able to provide any graphical modeling to facilitate the visualization of 4-dimensional spaces. Our proposed new multidimensional graphical modeling framework is missing from studies on Euclidian geometry and Minkowski's 4-dimensional spaces. The new multidimensional graphical modeling is based on the application of multidimensional physical spaces (Ruiz Estrada, 2011b).

\section{Introduction to the mega-space coordinate space}

We introduce the Mega-Space coordinate system, as shown in Figure 3. Universe $(\mathrm{U})$ is equivalent to the Mega-Space (S). The Mega-space Coordinate System is followed by the General-Spaces (GS), Sub-Spaces (SS), Micro-Spaces (MS), Nano-Spaces (NS) and JI-Spaces (see expression 18). The JI-Space applies formula (18) to plot each JI-Space into its Nano-Space. The JI-Space can be a negative or positive number in its Nano-Space coordinate system, but there is also a possibility that the number " 0 " is located at the center of all NanoSpace coordinate systems. Hence, the number " 0 " is a large surface located in the central part of all MicroSpaces that divides the positive and negative numbers of each JI-Space (see expression 19).

$\mathrm{U} \equiv \mathrm{S}=\left(\mathrm{GS}_{\mathrm{i},} \mathrm{SS}_{\mathrm{i}: \mathrm{j},} \mathrm{MS}_{\mathrm{i} \mathrm{ij}: \mathrm{k},} \mathrm{NS}_{\mathrm{i}: \mathrm{j}: \mathrm{k}: \mathrm{l},} \mathrm{JI}_{\mathrm{i}: \mathrm{j}: \mathrm{k}: \mathrm{l}: \mathrm{m}}\right)$

$\mathrm{L}=\alpha_{\mathrm{h}} \times \beta_{\mathrm{z}} / 2$

$M=\left[\left(X<i: j: k: \alpha_{h}>\right),\left(Y<i: j: k: \beta_{z}>\right)\right]$

Where $\mathrm{i}=\{1,2 \ldots \infty\} ; \mathrm{j}=\{1,2 \ldots \infty\} ; \mathrm{k}=\{1,2 \ldots \infty\} ; \mathrm{h}$ $=\{1,2 \ldots \infty\}$ and $\mathrm{z}=\{1,2 \ldots \infty\}$ 
Therefore, the Mega-Space Coordinate System starts from the General-Space "0", as shown in expression 21.

$\mathrm{U} \equiv \mathrm{S}=\mathrm{GS}_{0,} \mathrm{SS}_{0: 0,} \mathrm{MS}_{0: 0: 0,} \mathrm{NS}_{0: 0: 0: 0}, \mathrm{JI}_{0: 0: 0: 0: 0} \ldots$

Until the General-Space, the infinity Space $\infty \ldots$ is expressed by expression 22 .

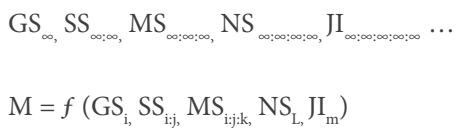

The final general function and all partial functions in analyzing the Mega-Space Coordinate System are equal to expressions 24, 25, 26, 27 and 28.

$$
\begin{aligned}
& \mathrm{S}=f\left(\mathrm{GS}_{0 \bigotimes} \mathrm{GS}_{1 \rrbracket \ldots} \mathrm{GS}_{\infty}\right) \\
& \mathrm{GS}=f\left(\mathrm{SS}_{0 \bigotimes} \mathrm{SS}_{1 \rrbracket} \mathrm{SS}_{\infty}\right) \\
& \mathrm{SS}=f\left(\mathrm{MS}_{0 \bigotimes} \mathrm{MS}_{1 \bigotimes \ldots} \mathrm{MS}_{\infty}\right) \\
& \mathrm{MS}=f\left(\mathrm{NS}_{0 \bigotimes} \mathrm{NS}_{1 \bigotimes \ldots} \mathrm{NS}_{\infty}\right) \\
& \mathrm{NS}=f\left(\mathrm{JI}_{0 \bigotimes} \mathrm{JI} \mathrm{I}_{1 \bigotimes \ldots} \mathrm{JI}_{\infty}\right)
\end{aligned}
$$

In fact, when we join all JI-Spaces located in different Nano-Spaces at the same Micro-Space, we observe a single surface in vertical position. Each JI-Space represents a single growth rate and constantly experiences different changes in different proportions compared with its neighbor JI-Spaces in the same Micro-Space. Hence, we can observe a large vertical surface constantly moving in its dimensions. We can determine that all Micro-Spaces move in their Sub-Spaces, that all Sub-Spaces move in their General-Spaces and, finally, that all General-Spaces move in the Mega-Spaces with variable speeds of time. It can be further stated that an economic phenomenon cannot be analyzed in isolation because every economic phenomenon is dynamic and complex by nature. Economic phenomena change dramatically and constantly and, therefore, will be studied from a multidimensional perspective. The traditional economic modeling approach is not able to precisely show the imperceptible changes associated with economic phenomena or behavior in the same graphical space. The multidimensional eco- nomic modeling framework can analyze any economic phenomenon from a multidimensional perspective to observe the unknown dimensions that cannot be observed with 2-dimensional modeling. Multidimensional economic modeling requires the use of graphical animation to generate real-time or slow motion visual effects to examine a giant graph move with different speeds of time live.

It will be useful to understand different scenarios that cannot be visualized using 2-dimensional modeling. Our final aim is to propose a powerful multidimensional economic and graphical modeling framework to observe different stages that any economic phenomenon can be experienced in real time or in slow motion.

\section{Definition of time in the mega-space coordinate system}

Our basic premise is that the Mega-Space or Universe is multidimensional. The JI-Space is a rigid body (value) that hangs in its Nano-Space. When all JI-Spaces are joined together, a surface in vertical position in the same Micro-Space can be generated. The Mega-space coordinate system applies three different types of time to its graphical modeling including general times (GT), partial times (PT) and constant times (KT), as previously mentioned.

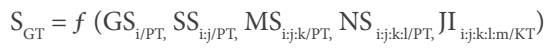

The Mega-Space runs on a general time, while General-Spaces, Sub-Spaces, Micro-Spaces and Nano-Spaces (see Figure 4) simultaneously run with partial times. The JI-Space hangs in its Micro-Space. A linear or non-linear curve in one Micro-Space can be obtained by joining the JI-Spaces. Expressions 30 and 31 clearly show that the Mega-Space Cartesian plane is applied in three different types of time, as previously mentioned.

$\mathrm{M}_{\mathrm{wt}}=f\left(\mathrm{GS}_{\mathrm{i} / \mathrm{wp},} \mathrm{SS}_{\mathrm{i:j} / \mathrm{wp},} \mathrm{MS}_{\mathrm{i}: \mathrm{j}: \mathrm{k} / \mathrm{wp},} \mathrm{NS}_{\mathrm{L} / \mathrm{wp},} \mathrm{JI}_{\mathrm{m} / \mathrm{wk}}\right)$

$\frac{\mathrm{L}_{\mathrm{wp}}=\alpha_{\mathrm{h} / \mathrm{wk}} \times \beta_{\mathrm{z} / \mathrm{wk}}}{2}$

Where

$\mathrm{h}=\{1,2 \ldots \infty\}$ and $\mathrm{z}=\{1,2 \ldots \infty\}$

$m_{w k}=\left[\left(X<i / w p: j / w p: k / w p: \alpha_{h / w k}>\right)\right.$, 

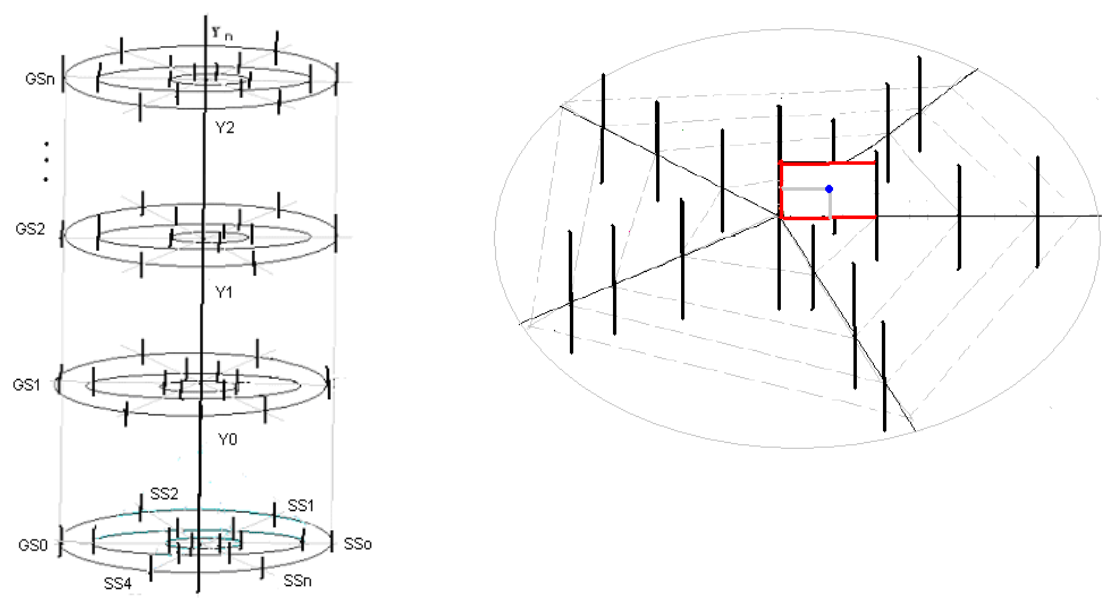

Figure 3. Mega-Space, General Space and Sub-Space (Ruiz Estrada, 2011b)

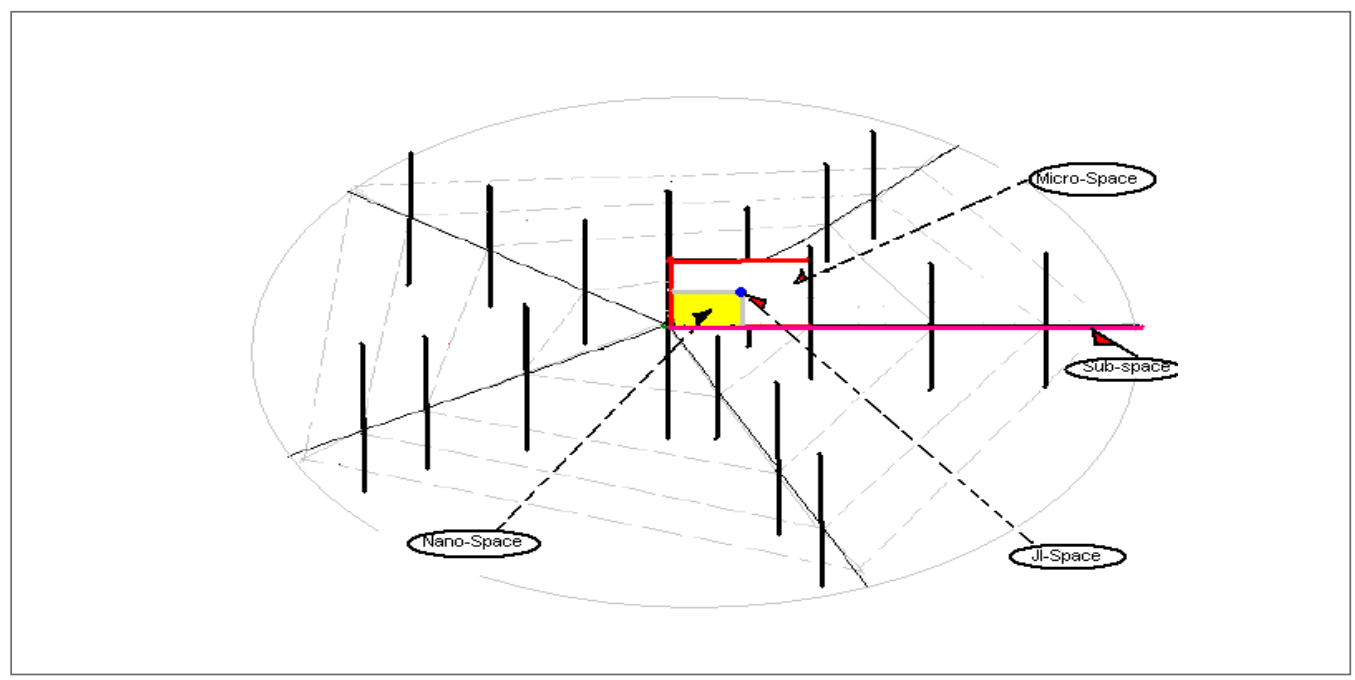

Figure 4. General-Space, Sub-Space, Micro-Space, Nano-Space and JI-Space (Ruiz Estrada, 2011 b)

$\left.\left(Y<\mathrm{i} / \mathrm{wp}: \mathrm{j} / \mathrm{wp}: \mathrm{k} / \mathrm{wp}: \beta_{z / w k}>\right)\right]$

Where

$\mathrm{i}=\{1,2 \ldots \infty\} ; \mathrm{j}=\{1,2 \ldots \infty\}$ and $\mathrm{k}=\{1,2 \ldots \infty\}$

How to plot on the mega-space cartesian plane

This section demonstrates how to plot on the Mega-
Space. Initially, we apply the Mega-Space Cartesian plane coordinate system that is shown by the following expression.

$\mathrm{U} \equiv \mathrm{M}=\left(\mathrm{GS}_{\mathrm{i},} \mathrm{SS}_{\mathrm{i}: \mathrm{j},} \mathrm{MS}_{\mathrm{i}: \mathrm{j}: \mathrm{k},} \mathrm{NS}_{\mathrm{L}, \mathrm{J}} \mathrm{JI}_{[(\mathrm{X}<\mathrm{i} \mathrm{j} ; \mathrm{j}: \mathrm{k}: \mathrm{ah}>,(\mathrm{Y}<\mathrm{i}: \mathrm{j}: \mathrm{k}: \beta z>)]}\right.$

$L=\underline{\alpha}_{\underline{h}} \times \beta_{\underline{z}} / 2$ 

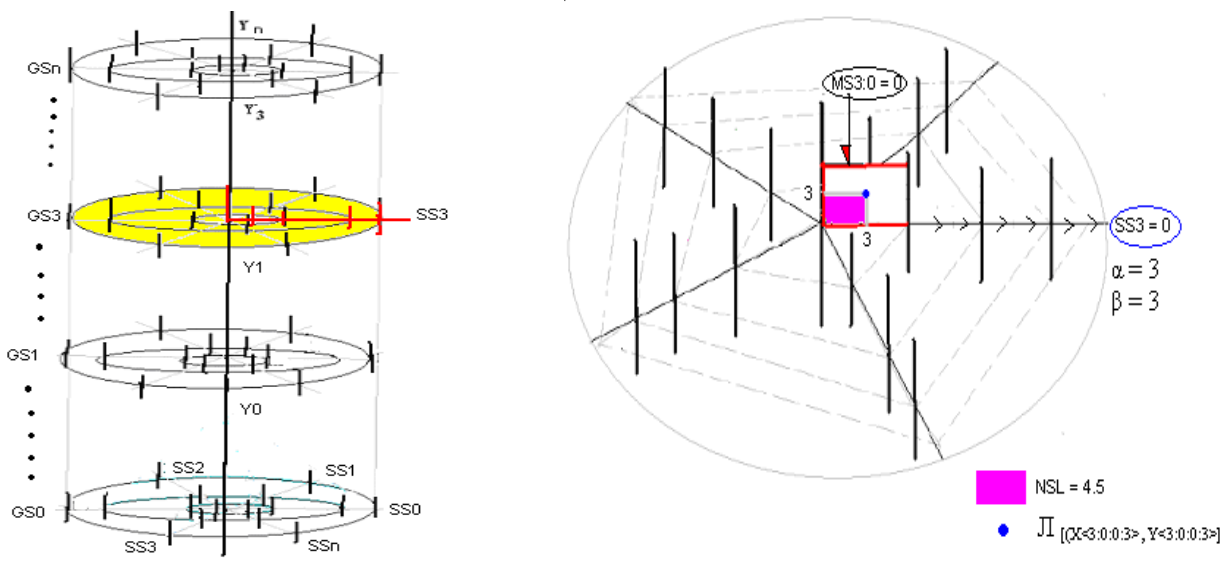

Figure 5. Mega-Space Plotting (Ruiz Estrada, 2011 b)

The values that should be used during the process of plotting on the Mega-Space Cartesian plane are shown in Figure 5.

$$
\begin{aligned}
& \mathrm{GS}_{\mathrm{i}}=3 \\
& \mathrm{SS}_{3}=0 \\
& \mathrm{MS}_{3: 0}=0 \\
& \mathrm{a}_{\mathrm{h}}=3 \\
& \beta_{\mathrm{z}}=3 \\
& \mathrm{NS}_{\mathrm{L}}=4.5 \\
& \left.\mathrm{JI}_{[(\mathrm{X}<3: 0: 0: 3>),(\mathrm{Y}<3: 0: 0: 3>)]}\right)
\end{aligned}
$$

\section{Introduction to the multidimensional real-time economic modeling framework}

Economic alerting modeling requires the creation of a data center that is able to save a large amount of information in different databases from different sources such as government agencies, academia, statistical departments and think tanks. The data center can also process a large amount of information in each database and simultaneously plot all the database information in a multidimensional coordinate system such as the mega-surface coordinate system. Similarly, we are also in a position to observe a mega-surface that is moving in real time through a large screen. Lastly, the database can alert us of possible failures in some or all the variables in one mega-surface at the same time. The main idea is to alert and generate special images in real time and slow motion. If we identify exactly which variable(s) is suffering, then we can take action(s) at the appropriate time to prevent more severe damage, for example, a possible major economic crisis in any economy. We assume that the mega-surface does not have a dependent variable; rather, it contains a large surface formed by infinite independent variables moving simultaneously. A megasurface moving continuously in relation to the different growth rates of independent variables can be generated by adding all these independent variables using straight lines. Furthermore, all these variables are assumed to be independent of each other. The purpose of generating the mega-surface is to study the behavior of several independent variables simultaneously in the same graphical space in real time. We can observe that it is possible to obtain high levels of accuracy and effectiveness to reduce the impact of a possible economic crisis in a timely manner. Our main focus is the ex-present effect in real time instead of the ex-post or ex-ante effect. The objective is to detect failure(s) in some or all independent variables in mega-surface live using the Omnia Mobilis assumption to relax all independent variables in our modeling and, thus, provide an alert of a possible economic crisis at any time. 


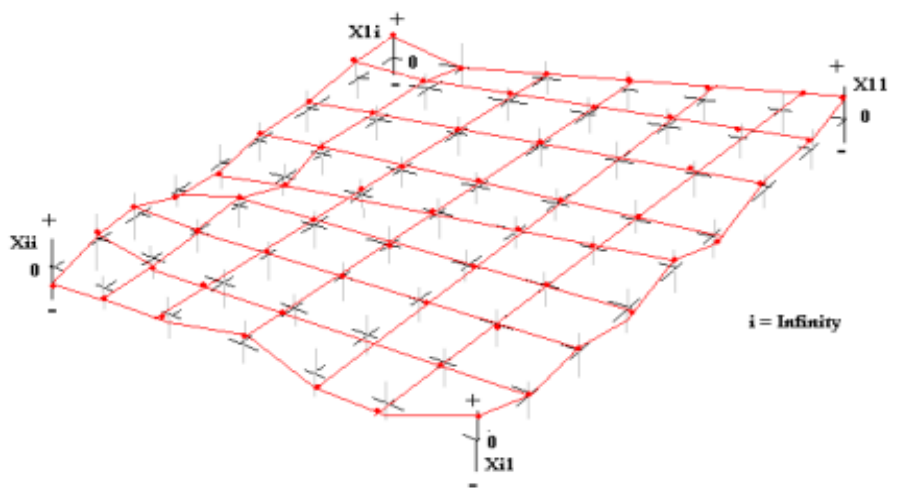

Figure 6. Mega-Surface (Ruiz Estrada, 2011b)

\section{Multidimensional economic modeling}

This paper proposes a multidimensional economic modeling framework that is able to generate a large number of simulations in the same graphical space in real time or slow motion to study the behavior of complex and dynamic economic phenomena. We suggest that multidimensional economic modeling requires the use of multidimensional physical spaces. The first assumption of multidimensional economic modeling is that all dimensions run under different speeds of time because any economic phenomenon can adopt a multidimensional behavior across time and space. The second assumption of multidimensional economic modeling is that any economic phenomenon always experiences a dynamic imbalanced state under the application of the Omnia Mobilis assumption. Hence, multidimensional economic modeling never needs to be in a state of equilibrium under the application of the Ceteris Paribus assumption.

Moreover, the idea of time in this model is rather different from that in 2-dimensional linear and nonlinear graphical modeling because 2-dimensional modeling refers to a single and unique time standard under the use of the traditional calendar (by months or years) to analyze complex and dynamic economic phenomena or behaviors. In our personal view, the 2-dimensional framework can clearly show analyti- cal inconsistency in the short and long runs. Therefore, we assume that any economic phenomenon can be analyzed from different dimensions because each dimension runs following a different speed of time. Furthermore, any economic phenomenon experiences different speeds of time. If we join different economic phenomena in the same graphical space, then it is possible to observe a series of economic phenomena moving in different directions with variable speeds of time in the same graphical space.

\section{Economic modeling in real time}

Economic modeling in real time can be done with the help of Econographicology (Ruiz Estrada, 2007). Econographicology supplies different multidimensional physical spaces to construct multidimensional graphs. The software required for economic modeling in real time follows various steps. First, there must be a standard format to input information on a line daily. Second, all these pieces of information (I) can be transferred to different databases (DB), which are interconnected to a unique information data center. Third, the same software can work immediately to plot different pieces of information (I) from different databases (DB) on the multidimensional physical space. Every observation from a database depends on different data sources such as the central bank, govern- 


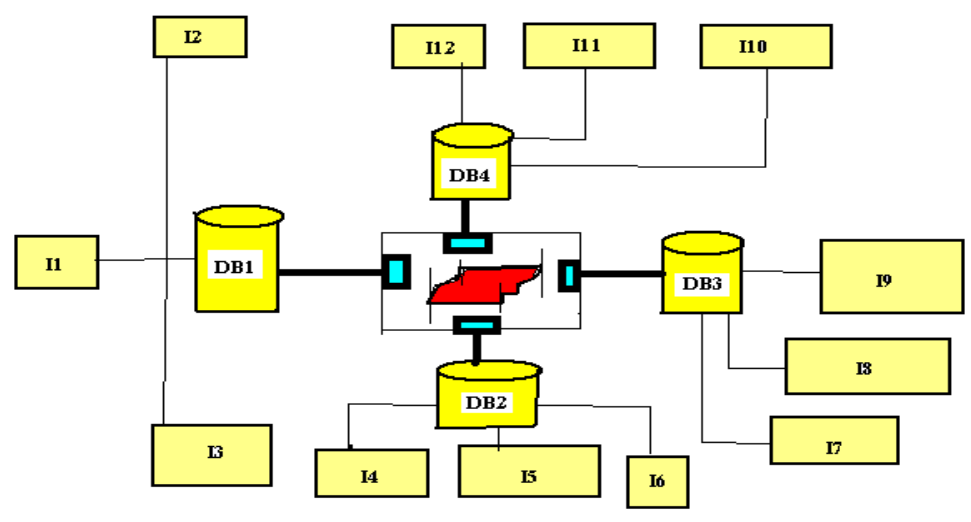

Figure 7. Economic Modeling in Real Time (Ruiz Estrada, 2011b)

ment agencies, private companies, national statistical departments and public and private research institutes, as demonstrated in Figure 6. Each plotted point in the multidimensional physical space is always changing. We use the concept of data changing in real time (see expression 37). Data changes in real time are due to the comparison of pieces of information (I) between two periods of time (the past and the present period of time). Similarly, the data changes in real time are fixed into their coordinate and variable positions all the time. Additionally, we reiterate that all changes in data in real time plotted in the multidimensional physical space are linked together with straight lines that form a single mega-surface in the same physical space (see Figure 6).

\section{Model}

Economic modeling in real time routinely starts with the input data function, as shown below:

$\mathrm{I}_{\mathrm{C}: \mathrm{R}}=\mathrm{Q}_{1}: \mathrm{Q}_{2}: \ldots: \mathrm{Q}_{\infty}$

$\mathbf{I}=$ Input answer $\mathbf{Q}=$ Question $(\mathrm{s}) \mathbf{C}=$ Column $\mathbf{R}=$ Row

The next step is the storing the data in the database (DB), which is described with the help of equation 36 .

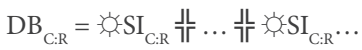

$\mathrm{C}=\{1,2,3 \ldots \mathrm{n}\} \mathrm{n}=\infty$

$\mathrm{R}=\{1,2,3 \ldots \mathrm{n}\} \mathrm{n}=\infty$

Where DB = Database, $\mathbf{C}=$ Column, $\mathbf{R}=$ Row, $=$ Running information in real time, SI = Save Information, and $\underset{\uparrow}{\Perp}=$ Interlink Database

In the case of data changes in real time ( $\left.-\Delta \mathrm{I}_{\mathrm{C}: \mathrm{R}}\right)$, we compare the information we received on the previous day ( $\mathbf{t}-\mathbf{1}=$ past period of time) and the information received on the current day ( $\mathbf{t}=$ actual period of time), as presented in expression 37.

$\Delta \mathrm{I}_{\mathrm{C}: \mathrm{R}}=\mathrm{SI}(\mathrm{t})-\mathrm{SI}(\mathrm{t}-1) /=\mathrm{SI}(\mathrm{t}-1)$

Finally, the plotting of real-time data is illustrated by expression 38, as follows:

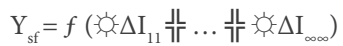

Next, Figure 7 further clarifies and simplifies the idea discussed above.

The multidimensional graphical signal detection model avoids the use of a single dependent variable. This model suggests replacing a single dependent variable with a single mega-surface. This mega-surface is formed by infinite axes, and each axis is located in a vertical position. An axis shows positive values on the top of the axis and negative values at the bottom of the axis 
(see Expression 36). Similarly, for each axis, it is possible to observe a point that is pending and moving up and down on its axis constantly. This point represents the behavior of an independent variable on its axis. Furthermore, we need to join each independent variable to its next independent variable neighbor using a straight line. Finally, we can observe that the mega-surface (see expression 37) is formed by joining all independent variables (see Figure 5). The mega-surface is always in constant movement in real time and moving in different directions. The real-time modeling effect generating into the mega-surface depends on the speed of the information reception that directly influences the behavior of each independent variable's growth rate (see Expression 39). We suggest the application of the Omnia Mobilis assumption to relax all independent variables that are moving on their axis in real time. Multidimensional graphical signal detection modeling starts by building the growth rate function of each independent variable, as shown by the following expression:

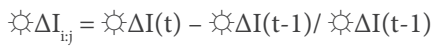

Where the row: $\mathrm{i}=\{1,2,3 \ldots \mathrm{n}\} \mathrm{n}=\infty$; the column: $\mathrm{j}$ $=\{1,2,3 \ldots \mathrm{n}\} \mathrm{n}=\infty ; \Delta=$ Growth rate; $=$ Running information in real time; $\mathrm{t}-1=$ past period of time and $\mathrm{t}=$ actual period of time. Each independent variable in the mega-surface in the coordinate system can be represented as below:

- $\Delta \mathrm{I}_{\mathrm{i}: \mathrm{j}}=\left(\mathrm{X}_{\mathrm{i}: \mathrm{j}} \mathrm{Y}_{\mathrm{i}: \mathrm{j}}\right)$

Where $\mathrm{X}=\{-\infty \ldots-3,-2,-1,0,1,2,3 \ldots \infty+\}$ and $\mathrm{Y}=$ $\{-\infty \ldots-3,-2,-1,0,1,2,3 \ldots \infty+\}$

The next step is to build the final mega-surface given by ( $i x j)$.

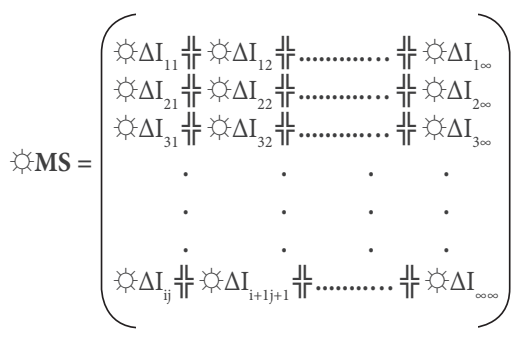

Where $i=\{1,2,3 \ldots \infty\} ; j=\{1,2,3 \ldots \infty\}$ and “L” is equal to the interlinking variables.
Finally, the analysis of the final mega-surface is based on the location of all independent variables in the mega-surface coordinate system. Hence, we obtain the following three possible results:

(1) If $\Delta \mathrm{I}_{\mathrm{ij}}>0$, then our mega-surface becomes GREEN in color, indicating good economic performance.

(2) If $\Delta \mathrm{I}_{\mathrm{ij}}=0$, then our mega-surface becomes YELLOW, showing that the economy is stagnant.

(3) If $\Delta \mathrm{I}_{\mathrm{ij}}<0$, then our mega-surface becomes RED, demonstrating that the economic performance of the economy is poor.

Put simply, the color of the mega-surface can alert us of a possible economic crisis in a timely manner. We would like to state that we cannot stop an economic crisis. Rather, we can only reduce the damage caused by an economic crisis. Therefore, the findings of this paper have serious implications for both researchers and policy makers.

\section{Conclusions}

This paper has attempted to develop an alternative modeling approach for researchers to utilize. The traditional 2-dimensional modeling approach is not able to explain complex and dynamic economic problems in real time. We have, therefore, attempted to develop an alternative economic modeling (MRTE-Modeling) framework that is 3-dimensional by construction. Similarly, we believe that alerting economic modeling is more effective than economic forecasting. Now, we are able to observe the behavior of any economic phenomenon as a whole in real time and the timing of possible market failures. However, it should also be noted that we cannot stop market failures; rather, we can only minimize their damage in the short and medium runs. Reducing the damages associated with market failures would certainly help policy makers make their economies more efficient by implementing and executing appropriate policies. The major contribution of this paper to the existing literature on macroeconomic modeling is that it provides solutions for various complex and dynamic economic phenomena in the same graphical space under the application of multi-times framework. We posit that any economic phenomenon needs to be studied from a multidimensional perspective and in real time 
based on the use of high-performing information technology and the application of multidimensional real time graphical modeling under Econographicology. The proposed modeling framework would enable researchers to observe the behavior of any economic phenomenon live through a screen and to find failures in the market in a timely manner. The idea behind applying multi-times framework is to generate a real-time effect or slow motion visual effect and to identify possible failures that any economic phenomenon can experience in different stages across time and space. Market behavior in the real world is always in a dynamic imbalance state. Therefore, the market does not necessarily need to be in a state of equilibrium in which only the price of the commodity (P) can influence the quantity demanded (Qd) and quantity supplied (Qs). It follows that the real-world market cannot be analyzed using only two variables to explain dynamic and complex market behaviors. In our opinion, the study of the market needs to include a large number of variables that affect demand and supply simultaneously. We, therefore, suggest the use of the price imbalance $(\mathrm{P} \approx)$ resulting from the relaxation or stress of all variables running into quantity demanded imbalance $(\mathrm{Qd} \approx)$ and the quantity supplied imbalance $(\mathrm{Q} \approx)$ ) simultaneously.

The proposed modeling framework is sufficiently flexible to include a large number of variables without any restriction or isolation. This is in contrast to the Ceteris Paribus assumption, which imposes restrictions and isolation into partial and general market equilibriums. A market basically consists of a large number of sub-markets in a dynamic imbalanced state. Therefore, if all sub-markets are kept in a dynamic imbalanced state, then the momentum of the imbalanced synchronization state can appear in different sub-markets simultaneously. The momentum of the imbalanced synchronization state is a result of the relaxation of all sub-markets. In fact, the momentum of the imbalanced synchronization state is a fleeting and unpredictable leak momentum that can appear spontaneously in all sub-markets at any time. Furthermore, the price commodity equilibrium $\left(\mathrm{P}^{*}\right)$ depends on the relaxation or stress of all the variables that affect the quantity demanded equilibrium $\left(\mathrm{Q}_{\mathrm{d}}{ }^{*}\right)$ and the quantity supplied equilib$\operatorname{rium}\left(\mathrm{Q}_{\mathrm{s}}^{*}\right)$.

\section{References}

Einstein, A. (1961). Relativity: The special and the general theory ( $3^{\text {rd }}$ ed.). New York, NY: Three Rivers Press.

Lafleur, L. (1960). Discourse on method, optics, geometry, and meteorology. New York, NY: The Liberal Arts Press.

Marshall, A. (1890). Principles of economics. London, UK: Macmillan and Co., Ltd.

McClelland, P. D. (1975). Causal explanation and model building in history, economics, and the new economic history. Ithaca, NY: Cornell University Press.

Merritt, F. D. (1898). Researches into the Mathematical Principles of the Theory of Wealth Augustin Cournot Nathaniel T. Bacon. Journal of Political Economy, 6 (3), 426-430.

Ruiz Estrada, M.A. (2007). Econographicology. International Journal of Economic Research, 4 (1), 75-86.

Ruiz Estrada, M. A., Yap, S. F., \& Nagaraj, S. (2008). Beyond the ceteris paribus assumption: modeling demand and supply assuming omnia mobilis. International Journal of Economic Research, 5 (2), 185-194.

Ruiz Estrada, M. A. (2011a). Policy modeling: definition, classification and evaluation. Journal of Policy Modeling, 33 (4), 523-536.

Ruiz Estrada, M. A. (2011b). Multi-dimensional coordinate spaces. International Journal of Physical Sciences, 6 (3), 340-357.

Ruiz Estrada, M. A., \& Yap, S. F. (2013). The origins and evolution of policy modeling. Journal of Policy Modeling, 35 (1), 170-182.

Schlicht, E. (1985). Isolation and aggregation in economics. Berlin: Springer-Verlag Publisher. 